\title{
Exosomes From Subjects With Multiple Sclerosis Express EBV-Derived Proteins and Activate Monocyte-Derived Macrophages
}

May F. Mrad, PhD, * Esber S. Saba, PhD, * Layane Nakib, MS, and Samia J. Khoury, MD

Neurol Neuroimmunol Neuroinflamm 2021;8:e1004. doi:10.1212/NXI.0000000000001004
Correspondence

Dr. Khoury

sk88@aub.edu.lb

\begin{abstract}
\section{Objective}

To investigate in a cross-sectional study the effect of serum-derived exosomes on primary human blood monocyte-derived macrophages (MDMs) comparing exosomes from healthy donors vs patients with relapsing-remitting multiple sclerosis in remission and in relapse and to assess whether the response correlates with exosomal Epstein-Barr virus (EBV) protein expression.
\end{abstract}

\section{Methods}

A total of 45 serum-derived exosome preparations were isolated from patients and healthy controls and verified for the expression of exosomal and EBV markers. MDMs were differentiated from monocytes for 7 days and incubated for 24 hours with exosomes, and then, cell supernatants were collected for cytokine measurement by cytometric bead array. Cells were immunophenotyped before and after differentiation.

\section{Results}

Serum-derived exosomes of patients with multiple sclerosis (MS) expressed higher levels of EBV proteins than healthy controls. Of interest, expression of EBV nuclear antigen EBNA1 and latent membrane proteins LMP1 and 2A was higher on exosomes derived from patients with active RRMS compared with healthy controls and stable patients. After data normalization, we observed that incubation with $\mathrm{EBV}(+)$ exosomes induced CXCL10 and CCL2 secretion by MDMs. MDMs differentiated from patients with active disease were better secretors of CXCL10 and other interferon- $\gamma$-inducible chemokines, including CCL2 and CXCL9, than MDMs from healthy and stable MS groups. MDMs from active patients had a higher frequency of a CD14(++) subset that correlated with the secreted CXCL10.

\section{Conclusion}

Exosomes expressing EBV proteins correlate with disease activity and induce an inflammatory response in MDMs that is compounded by the origin of the responder cells.

\footnotetext{
*These authors contributed equally to this work.

From the Nehme and Therese Tohme Multiple Sclerosis Center (M.F.M.), Faculty of Medicine, American University of Beirut Medical Center; Department of Experimental Pathology (E.S.S., L.N.), Immunology and Microbiology, Faculty of Medicine, American University of Beirut; and Nehme and Therese Tohme Multiple Sclerosis Center (S.J.K.), and Abu Haidar Neuroscience Institute, Faculty of Medicine, American University of Beirut Medical Center, Lebanon.

Go to Neurology.org/NN for full disclosures. Funding information is provided at the end of the article.

The Article Processing Charge was funded by the authors.
}

This is an open access article distributed under the terms of the Creative Commons Attribution-NonCommercial-NoDerivatives License 4.0 (CC BY-NC-ND), which permits downloading and sharing the work provided it is properly cited. The work cannot be changed in any way or used commercially without permission from the journal. 


\section{Glossary}

$\mathbf{B C L}=$ B-cell line; $\mathbf{C B A}=$ cytometric bead array; DMT = disease-modifying therapy; EBV = Epstein-Barr virus; IFN = interferon; $\mathbf{M D M}=$ monocyte-derived macrophage; $\mathbf{M S}=$ multiple sclerosis; $\mathbf{P B S}=$ phosphate-buffered saline; $\mathbf{P B M C}=$ peripheral blood mononuclear cell; RRMS = relapsing-remitting multiple sclerosis; VCA = viral capsid antigen.

Among the environmental risk factors for multiple sclerosis (MS), viral infections, specifically Epstein-Barr virus (EBV), have been highly associated with the disease. ${ }^{1}$ In fact, the vast majority of patients diagnosed with MS were found to be infected with EBV and had increasing titers of antibodies against the EBV nuclear antigen EBNA1 before the onset of neurologic symptoms. ${ }^{2,3}$ Moreover, studies on EBV protein expression in postmortem brains of patients with MS have revealed that a high proportion of $\mathrm{B}$ cells within the perivascular cuffs of active and chronic white matter lesions, as well as CNS-infiltrating plasma cells and CD8(+) T cells, express EBV proteins of latent and lytic phases. ${ }^{4,5}$ EBV may also infect microglia and astrocytes. ${ }^{6}$

Previous work has suggested a defect in CD8(+) T-cell reactivity to EBV in patients with MS. ${ }^{7,8}$ Of interest, adoptive transfer of autologous in vitro expanded EBV-specific CD8(+) T cells was used in a proof-of-concept study in a patient with progressive MS. ${ }^{9}$

Exosomes are important mediators of intercellular communication, mediating cell to cell signaling by carrying a wide range of molecules, and transferring them between cells. ${ }^{10}$ Their cargo includes proteins, lipids, mRNAs, noncoding RNAs, and miRNAs that can regulate gene expression and different signaling pathways in the recipient cells. ${ }^{10,11} \mathrm{EBV}$-associated exosomes pack a variety of viral components, including latent membrane proteins, EBV-encoded small RNAs, and miRNAs, which contribute to EBV infection and pathogenesis. ${ }^{12,13}$ In this study, we investigated the expression of EBV proteins in serum exosomes of patients with MS and controls and their effect on primary human blood monocyte-derived macrophages (MDMs). Because of the known associations between EBV infection and MS, our objective was to investigate whether EBV proteins are expressed in serum exosomes and whether they contribute to immune activation. Our results suggest that exosomes expressing EBV proteins correlate with disease activity and induce an inflammatory response in MDMs that is compounded by the origin of the responder cells.

\section{Methods}

\section{Study Population}

Patients and donors were recruited at the Nehme and Therese Tohme MS center as part of a longitudinal prospective cohort, the AUBMC-Multiple Sclerosis Interdisciplinary Research since 2012. This study was approved by research institutional review board (IM.SK1.08), and all participants provided written informed consent and assent as appropriate. For exosome marker profiling, we enrolled 30 patients classified as relapsingremitting multiple sclerosis (RRMS) according to the revised McDonald criteria. ${ }^{14}$ Subjects included stable RRMS $(\mathrm{n}=15)$, active RRMS (i.e., with a clinical relapse or gadoliniumenhancing lesions on MRI) $(n=15)$, and healthy controls $(n=$ 15). Responder cells were MDMs from peripheral blood mononuclear cells (PBMCs) of 10 healthy controls, 7 stable RRMS, and 7 active RRMS. Study population demographics and clinical characteristics are listed in table 1.

\section{Blood Processing, Exosome Isolation, and EBV Viral Capsid Antigen IgG Serology}

Peripheral blood was obtained by standard venipuncture and allowed to clot for 30 minutes followed by centrifugation at $800 \mathrm{~g}$ for 10 minutes at $4^{\circ} \mathrm{C}$, and serum was then collected and stored at $-80^{\circ} \mathrm{C}$. Exosomes were isolated from sera using total exosome isolation kit (Invitrogen) according to the manufacturer's instructions. EBV-infected B-cell line (BCL) and Hela exosomes were isolated from 48-hour serum-deprived cell supernatants using ultracentrifugation protocol as previously described. ${ }^{15}$ Exosome pellets were resuspended in phosphate-buffered saline (PBS), and protein content was measured using the Bio-Rad DC protein assay. Exosomes were stored in aliquots at $-80^{\circ} \mathrm{C}$. For EBV viral capsid antigen (VCA) IgG testing, sera samples were run in duplicates using Abbott ARCHITECT VCA IgG kit in the Clinical Chemistry laboratory at AUBMC.

\section{Scanning Electron Microscopy and Dynamic Light Scattering}

Ten microliter droplets of exosomes resuspended in $2 \%$ paraformaldehyde were applied and adsorbed on carbon-coated electron microscopy grids and then fixed with $50 \mu \mathrm{L}$ drop of $1 \%$ glutaraldehyde, according to a previously described protocol. ${ }^{15}$ The grids were then washed thoroughly in distilled water, dehydrated with an ascending ethanol sequence $(40 \%, 60 \%$, $80 \%$, and $97 \%$ ), left to dry, and then observed using TESCAN MIRA3 SEM at 5 and $20 \mathrm{kV}$. Alternatively, exosomes were diluted at different concentrations in PBS, filtered with $0.2-\mu \mathrm{m}$ pore size Minisart syringe filters, and measured on NanoPlus HD Particle Size Analyzer (Particulate Systems).

\section{Flow Cytometry of Exosomes}

One hundred fifty micrograms of exosomes was resuspended in PBS and incubated for 30 minutes with optimized concentrations of mouse monoclonal AF488-conjugated EBNA1 and AF647-conjugated LMP2 antibodies and rabbit polyclonal FITC-anticalnexin from Santa Cruz Biotechnology. 
Table 1 Exosome and Cell Donors' Demographics

\begin{tabular}{|c|c|c|c|}
\hline Characteristic & Healthy controls & Stable patients & Active patients \\
\hline \multicolumn{4}{|l|}{ Exosome set } \\
\hline No. & 15 & 15 & 15 \\
\hline Female & 9 & 9 & 10 \\
\hline Male & 6 & 6 & 5 \\
\hline Age (yrs), mean \pm SD & $34 \pm 12$ & $32 \pm 10$ & $37 \pm 13$ \\
\hline Disease duration (yrs), mean \pm SD & - & $6.4 \pm 5.5$ & $6.8 \pm 7.8$ \\
\hline EDSS score, mean \pm SD & - & $2.3 \pm 1.2$ & $2.6 \pm 1.9$ \\
\hline DMTs & None & None & $\begin{array}{l}\text { None }(9) \\
\text { Interferon beta-1 (5) } \\
\text { Fingolimod (1) }\end{array}$ \\
\hline \multicolumn{4}{|l|}{ PBMC set } \\
\hline No. & 10 & 7 & 7 \\
\hline Female & 6 & 4 & 5 \\
\hline Male & 4 & 3 & 2 \\
\hline Age (yrs), mean \pm SD & $35 \pm 8$ & $35 \pm 10$ & $36 \pm 13$ \\
\hline Disease duration (yrs), mean \pm SD & - & $6.8 \pm 5.9$ & $7.4 \pm 6.8$ \\
\hline EDSS score, mean \pm SD & - & $2.2 \pm 2$ & $2.4 \pm 1.4$ \\
\hline DMTs & None & $\begin{array}{l}\text { None (6) } \\
\text { Dimethyl fumarate (1) }\end{array}$ & $\begin{array}{l}\text { None (6) } \\
\text { Teriflunomide (1) }\end{array}$ \\
\hline
\end{tabular}

Abbreviations: DMTs = disease-modifying therapies; EDSS = Expanded Disability Status Scale; PBMC = peripheral blood mononuclear cell.

Labeled exosomes were then washed with PBS and ultracentrifuged at 100,000 $\mathrm{g}$ for 1 hour. Exosome acquisition was performed using specific flow cytometer settings and filter configurations. ${ }^{16,17}$ Briefly, the threshold was set to 200; forward and side scatter FSC/SSC parameters were set to log scale with both voltages set to $220 \mathrm{~V}$ and $250 \mathrm{~V}$, respectively. Acquisition was run at low flow rate $(\simeq 8 \mu \mathrm{L} /$ minute $)$, and 25,000 events were recorded for each sample. PBS and antibody alone in PBS were run for $>5$ minutes. Data acquisition and analysis were performed on a BD FACSAria SORP equipped with BD FACS Diva 6.0 software.

\section{Immunoblot Analysis}

Fifty micrograms of proteins was heated to $95^{\circ} \mathrm{C}$ in $2 \times$ Laemmli buffer (Bio-Rad), subjected to SDS-PAGE using mini-protean 4\%-15\% TGX Stain-Free gels (Bio-Rad), and then transferred to supported nitrocellulose membranes. Immunoblots were performed using mouse monoclonal CD63 and CD9, rabbit monoclonal TSG101 and LMP1 antibodies from Abcam, and rabbit polyclonal antibody against human calnexin $\mathrm{H}-70$ from Santa Cruz Biotechnology. This was followed by incubation with anti-rabbit or anti-mouse peroxidase secondary antibodies (Jackson ImmunoResearch). For CD9 and CD63 expression, SDS-PAGE was run under nonreducing conditions. Protein band signals were developed using enhanced chemiluminescence (Bio-Rad Clarity Western ECL Substrate).
Alternatively, immuno-dot blots of $5 \mu \mathrm{g}$ exosomes, spotted and adsorbed to nitrocellulose membranes, were performed using TSG101, CD63, and LMP1 antibodies. Pixels intensity of dots signals were quantified by ImageJ software.

\section{MDM Differentiation}

PBMCs, from whole-blood buffy coats isolated by Ficoll-density gradient centrifugation, were thawed and seeded at a concentration of $\simeq 2.5 \times 10^{6} \mathrm{cell} / \mathrm{mL}$ in a 6-well plate using X-VIVO 15 (Lonza). After adherence of monocytes, supernatants were aspirated, and complete RPMI 1640-Glutamax (Gibco), containing $10 \%$ fetal bovine serum and $1 \%$ penicillin/streptomycin, supplemented with $25 \mathrm{ng} / \mathrm{mL}$ M-CSF, was added. Cells were incubated at $5 \% \mathrm{CO}_{2}, 37^{\circ} \mathrm{C}$ humid incubator for 7 days

\section{Cell Treatment}

On day 7, MDMs were scraped and reseeded in flat-bottom 96-well plates at a density of $5 \times 10^{4}$ cell/well. Cells were treated for 24 hours with $25 \mu \mathrm{g}$ of serum exosomes, derived from 15 different healthy controls, 15 patients with stable RRMS, and 15 active patients. In all experiments, some wells were treated with $1 \mathrm{ng} / \mathrm{mL}$ LPS and $10 \mathrm{ng} / \mathrm{mL}$ interferon (IFN) $-\gamma$ as positive controls for induction of cytokine response and with BCL exosomes as an EBV-positive control. Exosome-free fluid, obtained after the final exosome pelleting, was used as negative control in optimization studies. Hela and 

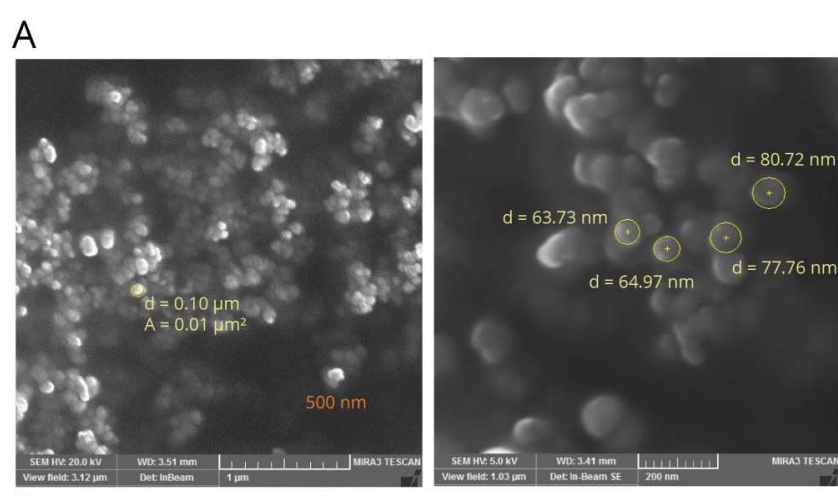

B

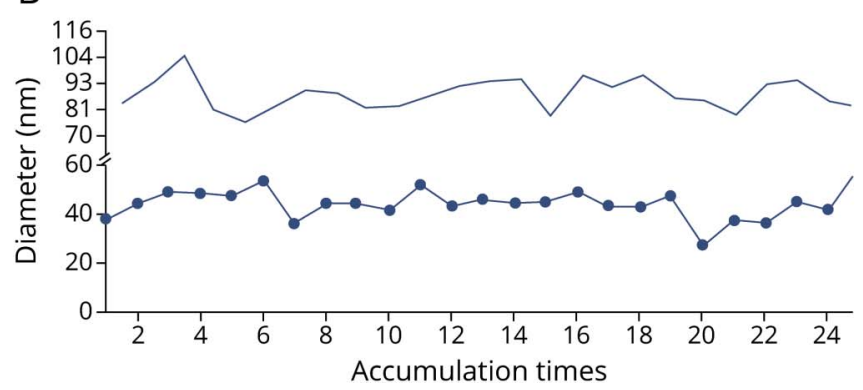

C
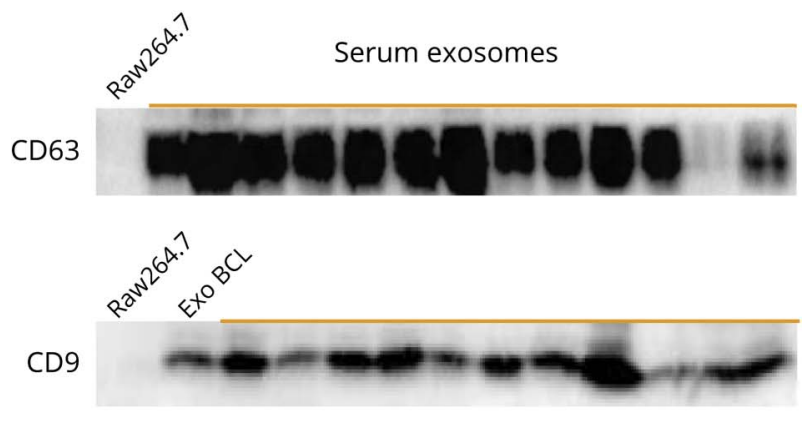

TSG101
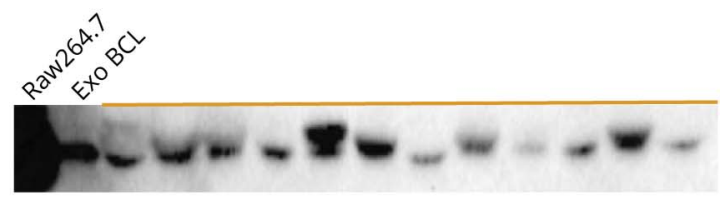

Calnexin

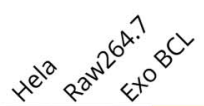

西

(A) Scanning electron microscope images showing exosome aggregates derived from sera of 2 donors ( 5 and $20 \mathrm{kV}$ ). Exosome diameter ranged from 50 to 120 $\mathrm{nm}$. (B) Dynamic light scattering of 2 representative exosome isolates. (C) Western blot of representative serum-derived exosomes showing expression of CD63, CD9, and TSG101 markers, but no detectable expression of calnexin. BCL exosomes were used as EBV-positive control and Raw 264.7 as EBV-negative control. Hela cell lysates were added as positive control for human calnexin IgG. BCL = B-cell line; EBV = Epstein-Barr virus; $\mathrm{MDM}=$ monocyte-derived macrophage.

Raw264.7 cell lines were used as EBV-negative control. In some experiments, MDMs were treated with exosomes that were labeled with PKH-26 red fluorescent cell linker (SigmaAldrich), according to the manufacturer's instructions. Cell fluorescence was acquired after 24 hours of treatment, using a Zeiss LSM 710 confocal microscope (Z-stacks, $0.2 \mu \mathrm{m}$ cell depth, $63 \times$ lens), or alternatively, by flow cytometry. For intracellular staining, cells were treated with exosomes in the presence of brefeldin A for 18 hours.

\section{Immunophenotyping for Monocyte/ Macrophage Cell Marker Expression}

Monocytes on day 1 (before differentiation) and MDMs on day 7 were stained with near-infrared live/dead stain (Invitrogen) and with multiple panels of antibodies, specified in table e-1, links.lww.com/NXI/A480. Cells were fixed and permeabilized using BD Cytofix/TF buffer set. Controls for multi-color flow cytometry staining included specific single antibody staining and compensation controls, as well as fluorescence minus one. Data were acquired on a BD FACSAria SORP. Analysis was subsequently performed using BD FACSDiva and R software. Mean fluorescence intensity (MFI) is presented on a logicle scale, as previously described. ${ }^{18}$

\section{Measurement of Secreted Cytokines}

Cell supernatants were collected 24 hours after treatment and stored at $-80^{\circ} \mathrm{C}$. Measurement of cytokines was performed using the BD Cytometric Bead Array (CBA) human soluble protein master buffer kit, following the manufacturer's instructions, for C-X-C motif chemokine ligand 10 (CXCL10, also known as interferon gamma-induced protein 10), interleukin 6, tumor necrosis factor $\alpha$, chemokine (C-C motif) ligand 2 (CCL2, otherwise known as monocyte chemoattractant protein-1/MCP-1), C-X-C motif chemokine ligand 9 (CXCL9, or monokine induced by gamma-interferon/ MIG), and chemokine (C-C motif) ligand 3 (CCL3, or macrophage inflammatory protein 1-alpha/MIP-1- $\alpha$ ). FCAP Array Software was used for signal quantification. Data were normalized to basal levels (subtracting the concentration of untreated cells).

\section{Statistical Analysis and Visualization}

For continuous variables, a Kruskal-Wallis followed by MannWhitney $U$ test for pairwise comparison was used. Data correlation was performed by the Pearson test. For categorical variables, the $\chi^{2}$ test was used. All data were analyzed using SPSS software Version 25. Differences in tests were considered statistically significant when $p<0.05$. To visualize cell subpopulations, individual flow cytometry standard (FCS) file from each run were combined into a single FCS file to define spatially distinct populations. Channels were first transformed using logicle transformation, and viable cells were selected from all events. Between 20,000 and 30,000 live cells were acquired. To eliminate sample size bias before performing 

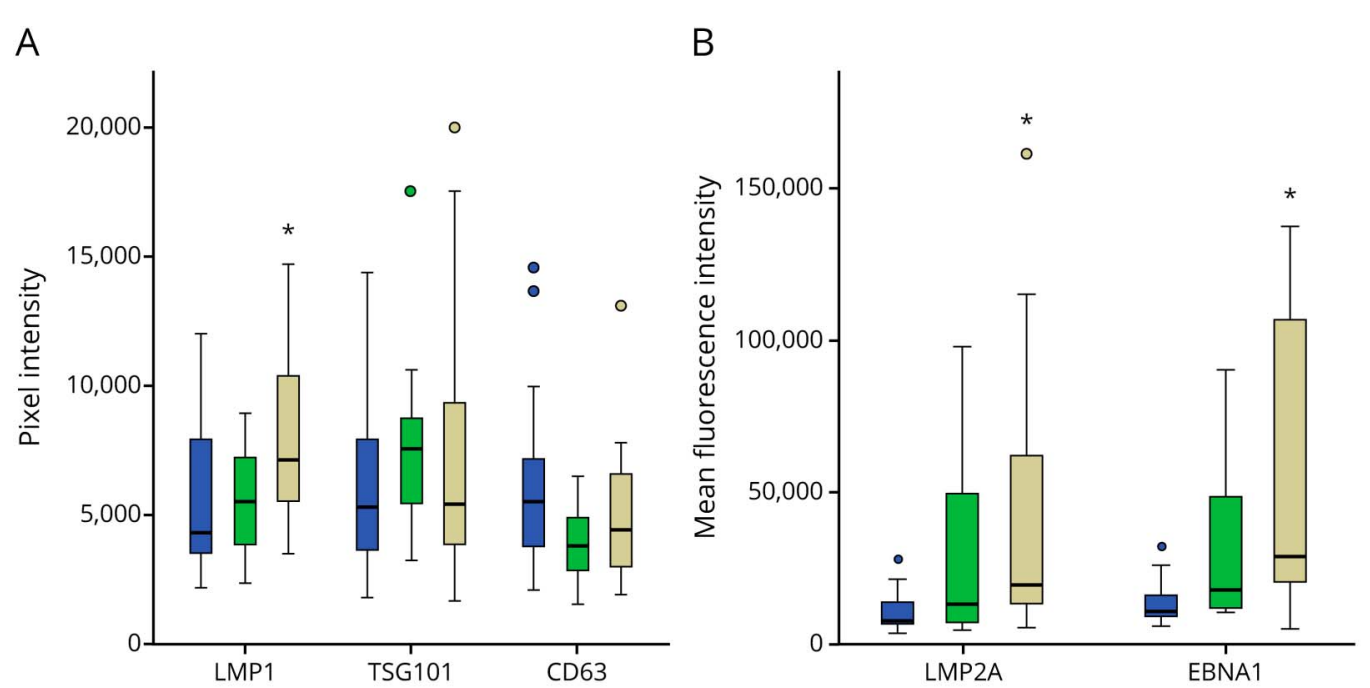

Exosome groups:

Healthy controls

Stable patients

Active patients

C

D

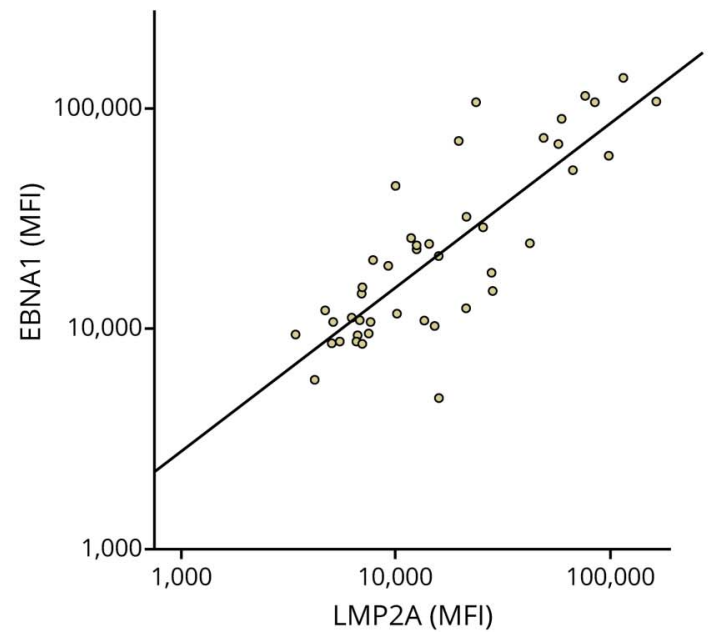

\begin{tabular}{cllccc}
\hline & & & \multicolumn{3}{c}{ EBV Cutoff } \\
& & & $\begin{array}{c}\text { Exosomes EBV } \\
\text { negative }\end{array}$ & $\begin{array}{c}\text { Exosomes EBV } \\
\text { positive }\end{array}$ & Total \\
\hline Groups Healthy & Count & 12 & 3 & 15 \\
& controls & $\%$ within disease & $80.0 \%$ & $20.0 \%$ & $100.0 \%$ \\
\cline { 2 - 6 } & Stable & Count & 7 & 8 & 15 \\
& patients & $\%$ within disease & $46.7 \%$ & $53.3 \%$ & $100.0 \%$ \\
\cline { 2 - 6 } & Active & Count & 5 & 10 & 15 \\
\multirow{4}{*}{ Total } & patients & $\%$ within disease & $33.3 \%$ & $66.7 \%$ & $100.0 \%$ \\
\cline { 2 - 5 } & & Count & 24 & 21 & 45 \\
& & $\%$ within disease & $53.3 \%$ & $46.7 \%$ & $100.0 \%$ \\
\hline
\end{tabular}

Exosomes from the sera of 15 healthy controls, 15 patients with stable RRMS, and 15 active patients were tested for the expression of exosome-related proteins and EBV-specific markers. (A and B) Boxplots showing pixel intensity and MFI of exosomes, tested for (A) LMP1, CD63, and TSG1 protein expression by immunoblots and for (B) LMP2A and EBNA1 by flow cytometry. MFI is presented on a logicle scale. Exosomes derived from active patients expressed significantly higher intensities of EBV-specific markers when compared with healthy controls. *Indicates a $p<0.05$. Exosome-specific markers did not show a significant distribution across tested groups. (C) Correlation curve of EBNA1 and LMP2A expression (MFI) on exosomes $(n=45, r=0.812$ ). (D) Distribution table of exosomes, per EBV cutoff, into EBV $(+)$ and $(-)$ groups. A cutoff was created using exosomal EBV mean MFI of a VCA-seronegative healthy control. EBV = Epstein-Barr virus; MDM = monocyte-derived macrophage; $M S$ = multiple sclerosis; RRMS = relapsing-remitting multiple sclerosis; VCA = viral capsid antigen.

dimensionality reduction, samples were randomly down sampled to 1,000 viable cells per sample, and analysis was run on equal numbers of events per sample. t-SNE is a nonlinear dimension reduction method that projects data into a lower dimensional space. ${ }^{19}$ Here, we applied t-SNE to compute the two-dimensional embedding of MDMs subtypes using the $\mathrm{R}$ package Rtsne. The implementation uses default parameters (iterations $=1,000$, perplexity $=30$, and $\theta=0.5$ ). Because we are interested in changes in the density of cell populations across different conditions, $\mathrm{t}$-SNE is followed by the unsupervised K-means clustering to cluster cells based on expression of different markers. K-means is a commonly used clustering algorithm for single-cell analysis after dimensionality reduction. ${ }^{20}$

\section{Data Availability}

All relevant data are provided in the article and supplementary files.

\section{Results}

\section{Exosome Characterization}

Exosomes were characterized according to the International Society for Extracellular Vesicles-recommended criteria. ${ }^{21}$ The size of isolated extracellular vesicles, ranging from $30-\mathrm{nm}$ diameter, typical for exosomes, was verified by electron microscopy (figure 1A). A similar size profile was confirmed by dynamic light scattering (figure 1B). These extracellular 
A

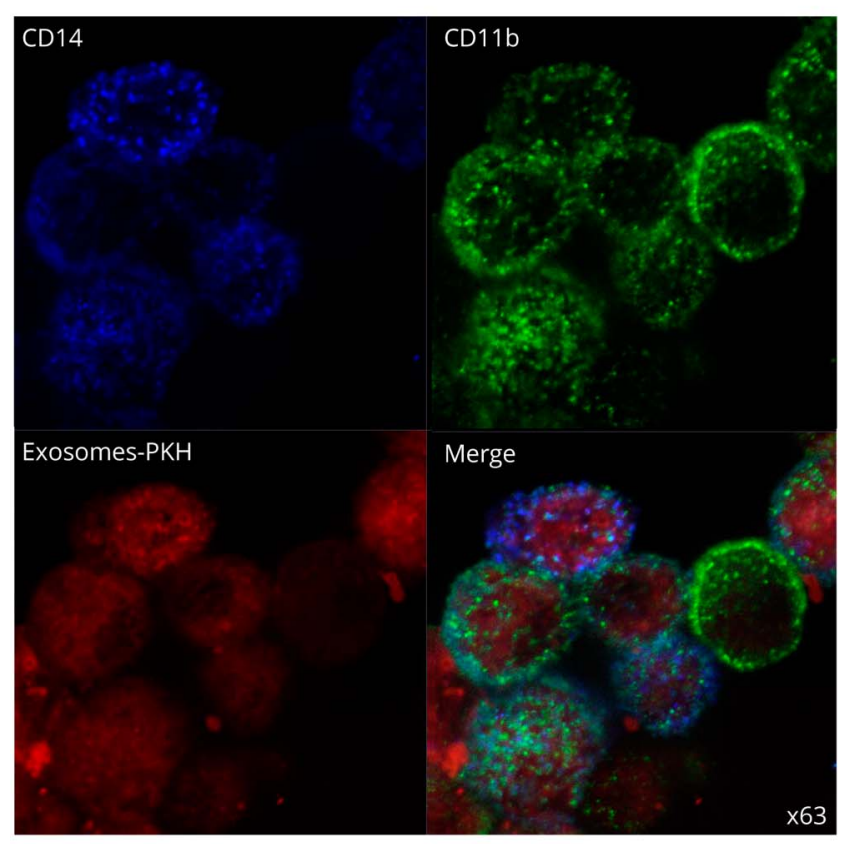

B

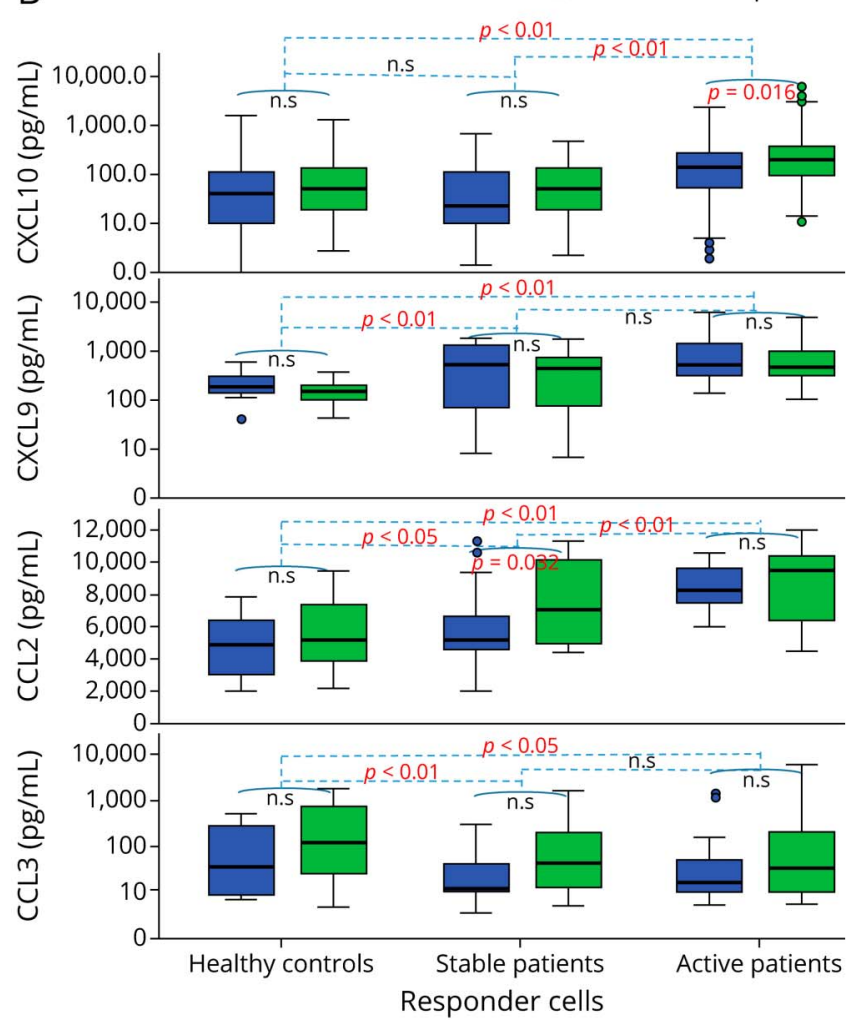

(A) Representative confocal microscopy images (single z-stacks) for MDMs after 24 hours of treatment with PKH-26-labeled exosomes (red). Cell-exosome uptake was confirmed with surface marker staining with V450-conjugated anti-CD14 and BB515-conjugated anti-CD11b. (B) Box plots of CXCL10, CCL2, CXCL9, and CCL3 secretion 24 hours after MDM treatment with exosomes, assessed using CBA. EBV(+) exosomes induced a higher CXCL10 secretion than EBV $(-)$ exosomes in cells of patients with active MS and a higher CCL2 secretion in cells of stable patients. A differential cytokine secretion was detected between MDMs of patients with MS and healthy controls. $n=24$ responder cells (10 healthy controls, 7 subjects with stable MS, and 7 subjects with active MS) for $C X C L 10$ and $n=12$ (4/group) for CXCL9, CCL2, and CCL3, each treated with 45 different exosomes (15/origin group). $p$ Values are indicated for comparison between exosome groups (EBV positive and negative) and for comparison between different responder cell groups (healthy, stable, and active MS cells). EBV = Epstein-Barr virus; MDM = monocyte-derived macrophage; MS = multiple sclerosis; n.s. = nonsignificant.

vesicles expressed exosome-enriched proteins CD63, CD9, and TSG101 with undetectable expression of the nonexosomal ER marker calnexin, as confirmed by Western blot analysis (figure 1C).

\section{Differential EBV Protein Expression by Exosomes From MS and Controls}

We have quantified using flow cytometry and dot blot the expression of several markers on exosomes (figure e-1, A and B, links.lww.com/NXI/A477). Some of the investigated markers are exosome specific like TSG101 and CD63, ${ }^{22}$ and others are related to EBV such as LMP1, LMP2A, and EBNA1. Exosome-specific markers, CD63 and TSG101, showed similar distribution across all groups (figure 2A). Exosomes from patients with active RRMS expressed higher EBV proteins when compared with exosomes of healthy controls (figure 2A, B). Stable RRMS exosomes tended to have a higher LMP2A expression compared with those from healthy controls; however, the difference did not reach statistical significance $(p=0.07)$. Furthermore, expression of LMP1, LMP2A, and EBNA1 was significantly correlated (figure e-2A, links.lww.com/NXI/A478, with a very strong correlation detected between LMP2A and EBNA1; $p=$ $0.0001, r=0.812$ ) (figure $2 \mathrm{C}$ ). All except 2 healthy individuals included in this study were EBV seropositive, with no significant differences in VCA IgG titers between groups (data not shown). There was no correlation between EBV serology and exosomal EBNA1 or LMP2A expression $(r=0.0006)$ (figure e-2B, links.lww.com/NXI/A478). Next, using the mean of LMP2A and EBNA1 MFI, we stratified exosomes into EBV-positive $(\operatorname{EBV}(+))$ and $\mathrm{EBV}$-negative $(\operatorname{EBV}(-))$ groups (figure 2D). The hypothetical cutoff was established using exosome derived from an EBV seronegative donor (by VCA IgG titers) (figure e-2B, links.lww.com/ NXI/A478).

Accordingly, we found that $\operatorname{EBV}(+)$ exosomes were those mostly derived from patients with RRMS. Almost all healthy control exosomes (80\%) fell below the cutoff level, thus into the $\operatorname{EBV}(-)$ group, whereas $57 \%$ of stable RRMS exosomes and $66 \%$ of active RRMS exosomes were $\operatorname{EBV}(+)$ (figure 2D) (Pearson $\chi^{2} p=0.009$ ). 

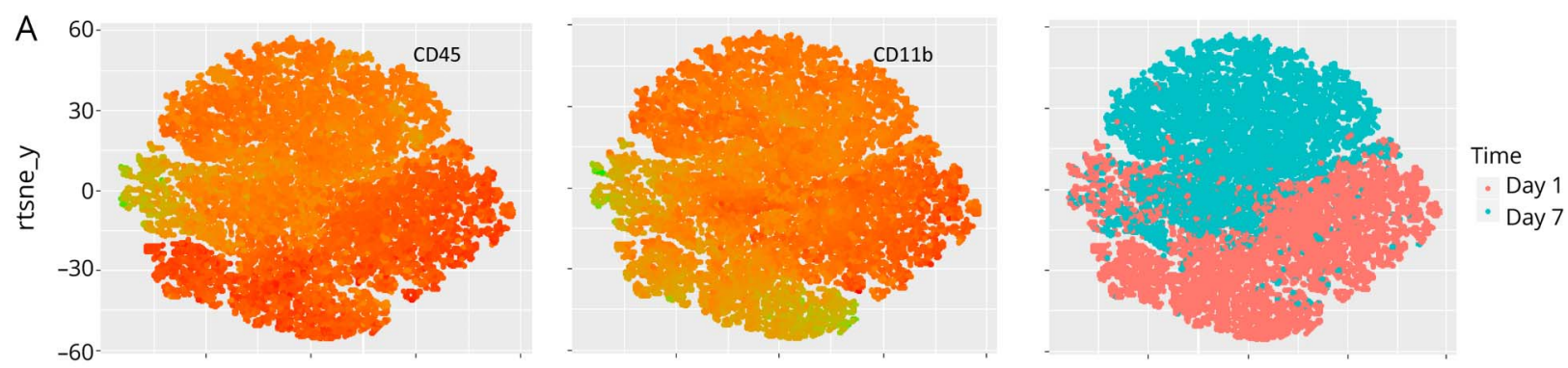

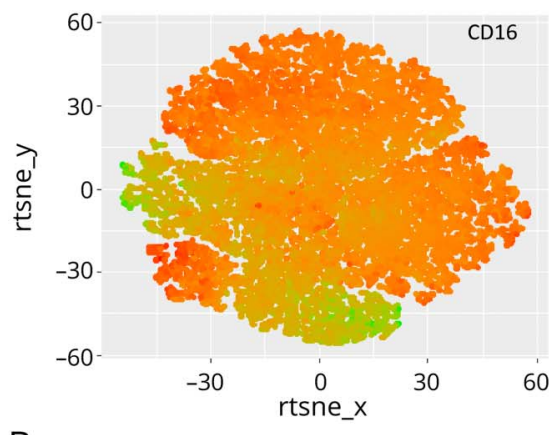

B.a Monocytes

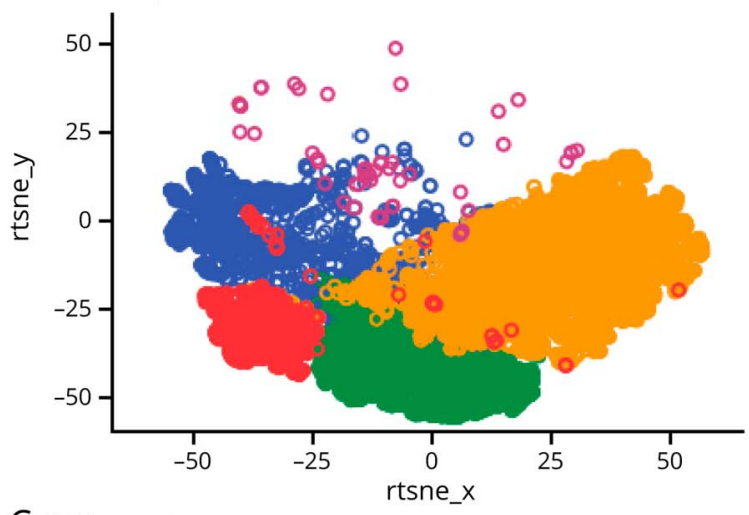

C.a Monocytes

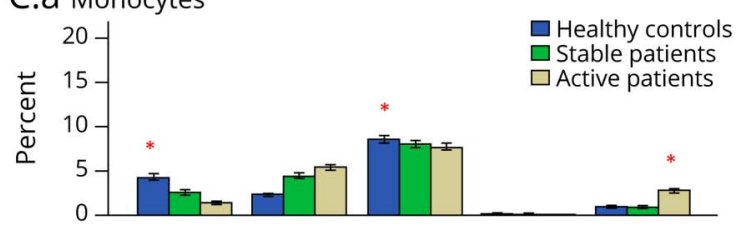

C.b Monocytes derived macrophages

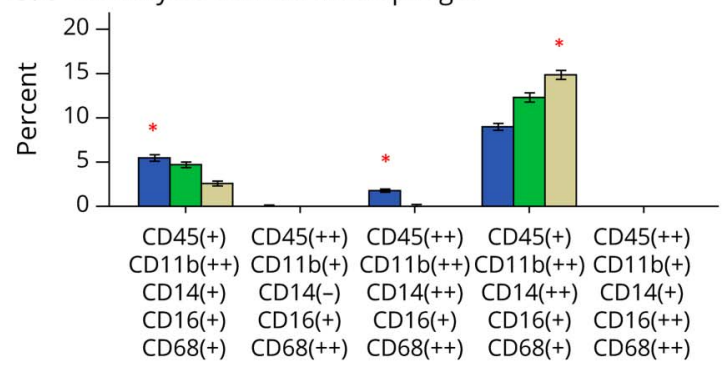

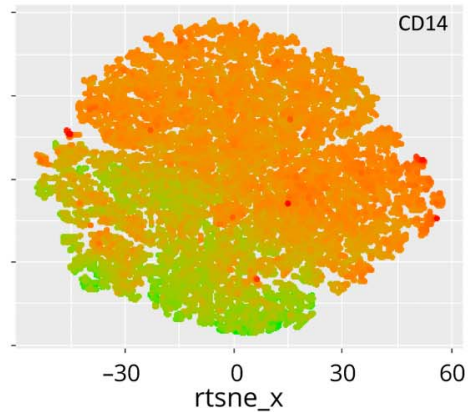

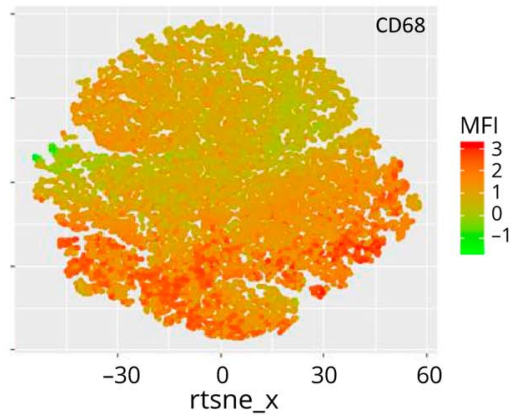

B.b Monocytes derived macrophages

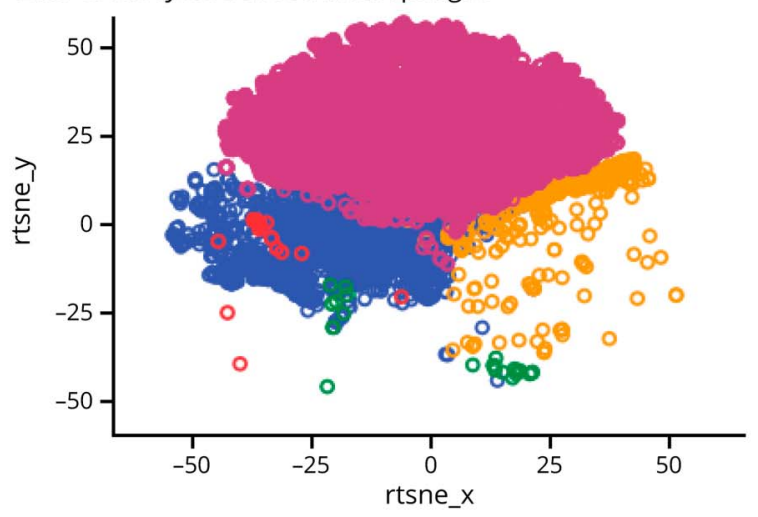

OCD45(+) CD11b(++) CD14(+) CD16(+) CD68(+) $\mathrm{OCD} 45(++) \mathrm{CD} 11 \mathrm{~b}(+) \mathrm{CD} 14(-) \mathrm{CD} 16(+) \mathrm{CD} 68(++)$ $\mathrm{OCD} 45(++) \mathrm{CD} 11 \mathrm{~b}(++) \mathrm{CD} 14(++) \mathrm{CD} 16(+) \mathrm{CD} 68(++)$ $\mathrm{OCD} 45(+) \mathrm{CD} 11 \mathrm{~b}(++) \mathrm{CD} 14(++) \mathrm{CD} 16(+) \mathrm{CD} 68(+)$ OCD45(++) CD11 b (+) CD14(+) CD16(++) CD68(++)

(A) t-SNE plots showing the distribution of different cell markers among monocytes and MDMs. The upper right panel indicates day 1 for clusters of monocytes/before differentiation (pink) and day 7 for MDMs/after differentiation (blue). The other panels show the distribution of expression intensities of single markers CD45, CD11b, CD14, CD16, and CD68 on the cellular clusters in relation to time, day 1 and day 7 . The color scale goes from green (low) to red (high) fluorescence intensity. Plots are representative of 12 different donors. (B) t-SNE showing the different monocytes and MDM subsets on days 1 and 7 , respectively. (C) Frequency (\%) of cell populations before and after differentiation from monocytes (day 1) to macrophages (day 7). (D) Pearson correlations of frequencies of different MDM subsets and chemokine secretion. Significant correlations $(p<0.05)$ are showing in red. $n=12$ independent experiments of 4 cell donors/group. MDM = monocyte-derived macrophage. ${ }^{*} p<0.05$ 


\section{EBV(+) Exosomes Induce CXCL10 Secretion by MDMs}

Next, we investigated the effect of serum exosomes on MDMs that were differentiated from monocytes of patients with MS and healthy controls. Exosome uptake by MDMs was verified, 24 hours after treatment, by confocal microscopy (figure 3A) and flow cytometry using $\mathrm{PKH}-26$ red fluorescent dye for exosome staining. Several cytokines were assessed in cells supernatants, CXCL10, CXCL9, CCL2, and CCL3 secretion showed certain dynamics upon treatment with exosomes compared with basal (untreated) levels. Of interest, EBV(+) exosomes induced a higher CXCL10 secretion than EBV(-) exosomes that reached significance when MDMs were derived from patients with active RRMS ( $p=0.016)$ (figure 3B). $\operatorname{EBV}(+)$ exosomes also induced a higher CCL2 secretion than EBV $(-)$ exosomes in cells of stable RRMS $(p=0.032)$. Furthermore, cells from active patients were more sensitive to exosome treatment independently of exosome phenotype (EBV positive or negative) or exosome origin (healthy or MS). These cells significantly secreted higher CXCL10 and CCL2 levels when compared with cells from healthy controls and stable patients and higher CXCL9 than healthy controls (figure 3B). MDMs of stable RRMS were also better secretors of CCL2 and CXCL9 relative to healthy controls. In contrast, CCL3 production was significantly higher in MDM cultures derived from healthy controls compared with patients with MS (figure 3B).

\section{Responder Cell Subsets in MDMs and Monocytes and Correlation With Response to Exosomes}

Responder cells were stained before and after differentiation using a panel of antibodies against CD45, CD11b, CD14, CD16, and CD68 to characterize immunophenotypic monocyte/macrophage subsets (table e-1, links.lww.com/ NXI/A480). On differentiation with M-CSF, cells acquired an elongated fibroblast-like morphology. Macrophages had an increased CD11b, CD16, and CD14 expression and a decreased CD45 and CD68 (figure 4A). High dimensionality reduction t-SNE followed by cluster analysis showed an enrichment of MDMs at day 7 toward 2 subsets CD45(+) $\mathrm{CD} 11 \mathrm{~b}(++) \mathrm{CD} 14(++) \mathrm{CD} 16(+) \mathrm{CD} 68(+)$ and $\mathrm{CD} 45(+)$ $\mathrm{CD} 11 \mathrm{~b}(++) \mathrm{CD} 14(+) \mathrm{CD} 16(+) \mathrm{CD} 68(+)$ (figure 4B). Of interest, the dominant $\mathrm{CD} 45(+) \mathrm{CD} 11 \mathrm{~b}(++) \mathrm{CD} 14(++)$ $\mathrm{CD} 16(+) \mathrm{CD} 68(+)$ population frequency showed a significant correlation with CXCL10 secretion, while a decrease in CD45(+)CD11b(++)CD14(+)CD16(+)CD68(+) population correlated with CXCL10, indicating a link between CXCL10 secretion and MDMs differentiation status (figure 4C, D). Furthermore, CXCL10 secretion was also correlated with the presence at baseline of a monocytic subset characterized by the following signature $\mathrm{CD} 45(++) \mathrm{CD} 11 \mathrm{~b}(+) \mathrm{CD} 14(+)$ CD16(++)CD68 $(++)$ at day $1(r=0.643, p=0.024)$. Whether the CXCL10 secretion reported at day 7 is linked to the presence of this specific type of cells is not known. However, this suggests that the difference seen in cells at day 1 may have favored the differentiation toward a better
CXCL10 secretor population at day 7. We then compared the proportion of these different subsets in each of our responder cell cultures; we found that the frequency of CD45(+)CD11b(++)CD14(++)CD16(+)CD68(+) subset was significantly higher in cells of patients with active RRMS compared with stable MS and healthy groups, whereas the frequency of CD45(+)CD11b(++)CD14(+)CD16(+) CD68(+) was significantly higher in the healthy controls cells (figure 4C). Our data suggest that MDM differentiation toward a CD45(+)CD11b(++)CD14(++)CD16(+) CD68(+) phenotype results in an enhanced CXCL10 secretion, after treatment with exosomes; however, the opposite was observed for CCL3 as this cytokine correlated with the CD45(+)CD11b(++)CD14(+)CD16(+)CD68(+) subset that was more enriched in the healthy control cells than MS cells (figure 4, C and D). There were no major differences between patients' MDMs and healthy MDMs for other myeloid cell markers including CD169/Siglec-1, CD206/macrophage mannose receptor, CD86, CD163, and HLADR (data not shown). The expression of HLADR, CD169, CD163, and CD86 on monocytic cells was highly retained on differentiation to MDMs. A mixed M1/M2 phenotype was identified in healthy and MS MDMs with a combined expression of CD206(+)CD163(+)CD86(+) phenotype. It is worth mentioning that the expression of CD206 was acquired on cell differentiation, whereas arginase- 1 was absent on both monocytes and differentiated macrophages on days 1 and 7, respectively.

\section{Discussion}

EBV infection is more common in patients with MS than in individuals without MS, and several reports support both a history of mononucleosis and high serum EBV antibody levels as MS disease risk factors. ${ }^{2,23}$ Exosomes bearing EBV markers are known to be released from EBV infected cells and in the circulation of EBV-infected individuals. ${ }^{13}$ But most of the available data on exosome expression of EBV markers originated from investigation performed on EBV-associated cancers. $^{24}$

Exosomes secreted from lymphoblastoid cell lines that contain LMP1 inhibit T-cell proliferation, ${ }^{25}$ and LMP1 peptides inhibit NK cell cytotoxicity. ${ }^{26}$ LMP1 may also modulate the selective sorting of proteins into the exosomal pathway; it has been reported that EBV-infected carcinoma cells secrete exosomes with a modified content and their proteomic analysis mapped to cellular activation pathways. ${ }^{27}$

To investigate the link between the epidemiologic findings and the immune response in patients with MS, we selected EBNA1 an essential protein for the replication and persistence of the viral episomes that is steadily expressed in all stages of EBV latencies and 2 membrane proteins, LMP1 and LMP2A, that are found in latency type II and $\mathrm{III}^{28}$ All the responder cells were from EBV-positive individuals, and there 
was no difference in serum VCA IgG titer between patients and controls. Our data show increased expression of EBV proteins in serum exosomes from patients with MS, specifically in patients with active disease, suggesting a change in dynamics of EBV protein expression associated with immune activation in MS.

In lymphoblastoid cell lines that secrete LMP1-containing exosomes, the LMP1 protein was found to be expressed in about $30 \%$ of the cells, but its expression was not correlated with the cell cycle. ${ }^{25}$ The authors speculated that LMP1 was in an intracellular reservoir and mimicked a constitutively active signaling molecule. ${ }^{25}$ Here, we focused on serum exosomes; thus, we do not know the source of the exosomes. We can hypothesize that EBV proteins are present in certain immune cells and their release into exosomes increases during periods of immune activation. Given that MDMs from patients with exacerbations are more sensitive to the effects of exosomes, we can speculate that they could have been sensitized in vivo. Whether $\operatorname{EBV}(+)$ exosomes initiate the immune activation during relapse or are a consequence of such activation remains to be investigated.

There are recent reports on RNAs and proteins content of exosomes in patients with $\mathrm{MS},{ }^{29,30}$ but no reports have tackled exosome EBV protein expression in MS. The exosomal cargo has functional consequences on MDMs, promoting the secretion IFN- $\gamma$-inducible cytokines. Here, we show that exosomes expressing EBV proteins induced CXCL10 and CCL2 secretion in cells of patients with RRMS. Of interest, MDMs from active patients were more sensitive to exosomes compared with cells from stable patients and healthy controls and secreted the highest amounts of CXCL10 and other IFN- $\gamma$-inducible chemokines CCL2 and CXCL9. Nevertheless, these cells secreted significantly less CCL3 when compared with cells from stable patients and healthy controls. The observed response could be related to exosomes' cargo, to MDMs' differentiation status, or to a combination of both.

Enhanced chemokine expression was described within active demyelinating MS lesions, predominantly by macrophages/ microglial cells and reactive astrocytes. ${ }^{31}$ Recent single-cell RNA-seq analyses identified a signaling cascade driven by monocytes expressing CXCL9 and CXCL10 that together with NK promotes Th1 responses in pathogen-exposed mice lymph nodes. ${ }^{32}$ Lately, Giladi et al. ${ }^{33}$ described CXCL10(+) monocytes as a pathogenic subset in the CNS of EAE mice. Elevated levels of CXCL10 and other chemokines including CCL2, CCL3, and CXCL9 were also reported in MS CSF and serum, ${ }^{34,35}$ despite discrepancies in data regarding levels of CCL2 and CCL $3 .^{36}$ These chemokines vary in expression in different phases of $\mathrm{MS}^{34}$ and are differentially modulated by disease-modifying therapies (DMTs), yet the data are inconclusive or conflicting in that regard. ${ }^{35,37}$ Different modes of cellular exosome uptake have been described ${ }^{11}$ that is highly dependent on the nature of recipient cells rather than the exosomal molecules. ${ }^{38}$ Here, we have shown that exosomes are internalized within multiple MDM subsets, and the subsets highly expressing $\mathrm{CD} 14(++)$ were more responsive to treatment.

Our data are in line with previous reports describing nonclassical CD14(+)CD16(+) phenotypes in circulating monocytes of patients with MS, and its association with elevated expression of proinflammatory cytokines, ${ }^{39}$ and with data suggesting that $\mathrm{CD} 16(+)$ monocytes facilitate T-cell migration to the CNS and preferentially activate Th17 cells. $^{40}$

Limitations of this study include the relatively small number of responder cell donors and the cross-sectional design, so future studies should include longitudinal evaluation of exosomes. Another possible limitation is the inclusion of a few patients with MS on treatment raising the possibility that DMTs can alter the outcome of the functional assays. However, we show that exosomes from treated or untreated patients had similar expression of EBV proteins (figure e-3A, links.lww.com/NXI/A479), and their effects on CXCL10 production by MDMs were the same (figure e-3, B and C, links.lww.com/NXI/A479), thus mitigating the possible interference by DMTs.

This study contributes to our understanding of the association between EBV and MS risk. EBV $(+)$ exosomes are increased in the serum of patients with MS in relapse that promote proinflammatory response by antigen-presenting cells and may contribute to the ongoing immune activation. Further work is needed to clarify whether reactivation of EBV drives MS disease activation or whether it is just a consequence of immune activation.

\section{Acknowledgment}

The authors thank Dr. Aida Habib for providing equipment and reagents for immunoblots and Dr. Marwan El-Sabban for technical assistance in confocal microscopy.

\section{Study Funding}

This study was funded by the Lebanese National Council for Scientific Research (LNCSR).

\section{Disclosure}

M.F. Mrad, E.S. Saba, and Layane Nakib report no disclosures. S.J. Khoury declares receiving investigator-initiated grant support from Novartis. She also served on a scientific advisory board for Merck and served on a DSMB for Biogen. She serves as an associate editor for Annals of Neurology and section editor for Clinical Immunology. Go to Neurology.org/NN for full disclosures.

\section{Publication History}

Received by Neurology: Neuroimmunology \& Neuroinflammation October 30, 2020. Accepted in final form February 26, 2021. 
Appendix Authors

\begin{tabular}{|c|c|c|}
\hline Name & Location & Contribution \\
\hline $\begin{array}{l}\text { May F. } \\
\text { Mrad, } \\
\text { PhD }\end{array}$ & $\begin{array}{l}\text { Nehme and Therese Tohme } \\
\text { Multiple Sclerosis Center at the } \\
\text { American University of Beirut } \\
\text { Medical Center, Lebanon }\end{array}$ & $\begin{array}{l}\text { Study concept and design; data } \\
\text { acquisition; data analysis and } \\
\text { interpretation; drafting of text } \\
\text { and figures; and revising the } \\
\text { manuscript for intellectual } \\
\text { content }\end{array}$ \\
\hline $\begin{array}{l}\text { Esber S. } \\
\text { Saba, } \\
\text { PhD }\end{array}$ & $\begin{array}{l}\text { Department of Experimental } \\
\text { Pathology, Immunology and } \\
\text { Microbiology at the American } \\
\text { University of Beirut, Lebanon }\end{array}$ & $\begin{array}{l}\text { Statistical analysis; data } \\
\text { analysis and interpretation; } \\
\text { drafting of text and figures; and } \\
\text { revising the manuscript for } \\
\text { intellectual content }\end{array}$ \\
\hline $\begin{array}{l}\text { Layane } \\
\text { Nakib, } \\
\text { MS }\end{array}$ & $\begin{array}{l}\text { Department of Experimental } \\
\text { Pathology, Immunology and } \\
\text { Microbiology at the, American } \\
\text { University of Beirut, Lebanon }\end{array}$ & $\begin{array}{l}\text { Data acquisition; data analysis } \\
\text { and interpretation; and } \\
\text { revising the manuscript }\end{array}$ \\
\hline $\begin{array}{l}\text { Samia J. } \\
\text { Khoury, } \\
\text { MD }\end{array}$ & $\begin{array}{l}\text { Abu Haidar Neuroscience } \\
\text { Institute and Nehme and } \\
\text { Therese Tohme Multiple } \\
\text { Sclerosis Center at the } \\
\text { American University of Beirut } \\
\text { Medical Center, Lebanon }\end{array}$ & $\begin{array}{l}\text { Conceptualization; funding } \\
\text { acquisition; data analysis and } \\
\text { interpretation; and revising the } \\
\text { manuscript for intellectual } \\
\text { content }\end{array}$ \\
\hline
\end{tabular}

\section{References}

1. Ascherio A, Munger KL. Environmental risk factors for multiple sclerosis. Part I: the role of infection. Ann Neurol. 2007;61:288-299.

2. Langer-Gould $\mathrm{A}, \mathrm{Wu} \mathrm{J}$, Lucas $\mathrm{R}$, et al. Epstein-Barr virus, cytomegalovirus, and multiple sclerosis susceptibility: a multiethnic study. Neurology. 2017;89:1330-1337.

3. Abrahamyan S, Eberspacher B, Hoshi MM, et al. Complete Epstein-Barr virus seropositivity in a large cohort of patients with early multiple sclerosis. J Neurol Neurosurg Psychiatry. 2020;91:681-686.

4. Moreno MA, Or-Geva N, Aftab BT, et al. Molecular signature of Epstein-Barr virus infection in MS brain lesions. Neurol Neuroimmunol Neuroinflamm. 2018;5:e466.

5. Serafini B, Rosicarelli B, Veroni C, Mazzola GA, Aloisi F. Epstein-Barr virus-specific $\mathrm{CD} 8 \mathrm{~T}$ cells selectively infiltrate the brain in multiple sclerosis and interact locally with virus-infected cells: clue for a virus-driven immunopathological mechanism. J Virol. 2019;93:e00980-e009819.

6. Hassani A, Corboy JR, Al-Salam S, Khan G. Epstein-Barr virus is present in the brain of most cases of multiple sclerosis and may engage more than just B cells. PLoS One. 2018;13:e0192109.

7. Pender MP, Csurhes PA, Lenarczyk A, Pfluger CM, Burrows SR. Decreased T cell reactivity to Epstein-Barr virus infected lymphoblastoid cell lines in multiple sclerosis. J Neurol Neurosurg Psychiatry. 2009;80:498-505.

8. Pender MP, Csurhes PA, Burrows JM, Burrows SR. Defective T-cell control of Epstein-Barr virus infection in multiple sclerosis. Clin Transl Immunol. 2017;6:e126.

9. Pender MP, Csurhes PA, Smith C, et al. Epstein-Barr virus-specific adoptive immunotherapy for progressive multiple sclerosis. Mult Scler. 2014;20:1541-1544.

10. Robbins PD, Morelli AE. Regulation of immune responses by extracellular vesicles. Nat Rev Immunol. 2014;14:195-208.

11. Mathieu M, Martin-Jaular L, Lavieu G, Thery C. Specificities of secretion and uptake of exosomes and other extracellular vesicles for cell-to-cell communication. $\mathrm{Nat} \mathrm{Cell}$ Biol. 2019;21:9-17.

12. Meckes DG Jr. Exosomal communication goes viral. J Virol. 2015;89:5200-5203.

13. Baglio SR, van Eijndhoven MA, Koppers-Lalic D, et al. Sensing of latent EBV infection through exosomal transfer of 5'pppRNA. Proc Natl Acad Sci USA. 2016;113:E587-E596.
14. Thompson AJ, Banwell BL, Barkhof F, et al. Diagnosis of multiple sclerosis: 2017 revisions of the McDonald criteria. Lancet Neurol. 2018;17:162-173.

15. Thery C, Amigorena S, Raposo G, Clayton A. Isolation and characterization of exosomes from cell culture supernatants and biological fluids. Curr Protoc Cell Biol. 2006.Chapter 3:Unit 3.22.

16. Inglis HC, Danesh A, Shah A, Lacroix J, Spinella PC, Norris PJ. Techniques to improve detection and analysis of extracellular vesicles using flow cytometry. Cytometry A. 2015;87:1052-1063.

17. Saenz-Cuesta M, Arbelaiz A, Oregi A, et al. Methods for extracellular vesicles isolation 381 in a hospital setting. Front Immunol. 2015;6:50

18. Parks DR, Roederer M, Moore WA. A new "Logicle" display method avoids deceptive effects of logarithmic scaling for low signals and compensated data. Cytometry A. 2006; 69:541-551.

19. van der Maaten L, Hinton G. Visualizing data using t-SNE. J Machine Learn Res. 2008; 9:2579-2605.

20. Grun D, Lyubimova A, Kester L, et al. Single-cell messenger RNA sequencing reveals rare intestinal cell types. Nature. 2015;525:251-255.

21. Lotvall J, Hill AF, Hochberg F, et al. Minimal experimental requirements for definition of extracellular vesicles and their functions: a position statement from the International Society for Extracellular Vesicles. J Extracell Vesicles. 2014;3:26913.

22. Yoshioka Y, Konishi Y, Kosaka N, Katsuda T, Kato T, Ochiya T. Comparative marker analysis of extracellular vesicles in different human cancer types. J Extracell Vesicles. 2013;2.doi:10.3402/jev.v2i0.20424.

23. Pakpoor J, Ramagopalan SV. Epstein-Barr virus is a necessary causative agent in the pathogenesis of multiple sclerosis: yes. Mult Scler. 2013;19:1690-1691.

24. Teow SY, Liew K, Khoo AS, Peh SC. Pathogenic role of exosomes in Epstein-Barr virus (EBV)-Associated cancers. Int J Biol Sci. 2017;13:1276-1286.

25. Flanagan J, Middeldorp J, Sculley T. Localization of the Epstein-Barr virus protein LMP 1 to exosomes. J Gen Virol. 2003;84:1871-1879.

26. Dukers DF, Meij P, Vervoort MB, et al. Direct immunosuppressive effects of EBVencoded latent membrane protein 1. J Immunol. 2000;165:663-670.

27. Meckes DG Jr, Gunawardena HP, Dekroon RM, et al. Modulation of B-cell exosome proteins by gamma herpesvirus infection. Proc Natl Acad Sci USA. 2013;110: E2925-E2933.

28. Zhao M, Nanbo A, Sun L, Lin Z. Extracellular vesicles in Epstein-Barr virus' life cycle and pathogenesis. Microorganisms. 2019;7:48.

29. Ebrahimkhani S, Vafaee F, Young PE, et al. Exosomal microRNA signatures in multiple sclerosis reflect disease status. Sci Rep. 2017;7:14293.

30. Selmaj I, Cichalewska M, Namiecinska M, et al. Global exosome transcriptome profiling reveals biomarkers for multiple sclerosis. Ann Neurol. 2017;81:703-717.

31. Mayo L, Quintana FJ, Weiner HL. The innate immune system in demyelinating disease. Immunol Rev. 2012;248:170-187.

32. Blecher-Gonen R, Bost P, Hilligan KL, et al. Single-cell analysis of diverse pathogen responses defines a molecular roadmap for generating antigen-specific immunity. Cell Syst. 2019;8:109-121.e106.

33. Giladi A, Wagner LK, $\mathrm{Li} \mathrm{H}$, et al. Cxcl10(+) monocytes define a pathogenic subset in the central nervous system during autoimmune neuroinflammation. Nat Immunol. 2020;21:525-534.

34. Charo IF, Ransohoff RM. The many roles of chemokines and chemokine receptors in inflammation. N Engl J Med. 2006;354:610-621.

35. Huang J, Khademi M, Fugger L, et al. Inflammation-related plasma and CSF biomarkers for multiple sclerosis. Proc Natl Acad Sci USA. 2020;117:12952-12960.

36. Kothur K, Wienholt L, Brilot F, Dale RC. CSF cytokines/chemokines as biomarkers in neuroinflammatory CNS disorders: a systematic review. Cytokine. 2016;77: 227-237.

37. Bai Z, Chen D, Wang L, et al. Cerebrospinal fluid and blood cytokines as biomarkers for multiple sclerosis: a systematic review and meta-analysis of 226 studies with 13,526 multiple sclerosis patients. Front Neurosci. 2019;13:1026.

38. Horibe S, Tanahashi T, Kawauchi S, Murakami Y, Rikitake Y. Mechanism of recipient cell-dependent differences in exosome uptake. BMC Cancer. 2018;18:47.

39. Chuluundorj D, Harding SA, Abernethy D, La Flamme AC. Expansion and preferential activation of the CD14(+)CD16(+) monocyte subset during multiple sclerosis. Immunol Cell Biol. 2014;92:509-517.

40. Waschbisch A, Schroder S, Schraudner D, et al. Pivotal role for CD16+ monocytes in immune surveillance of the central nervous system. J Immunol. 2016;196:1558-1567. 


\title{
Neurology \\ Neuroimmunology \& Neuroinflammation
}

\author{
Exosomes From Subjects With Multiple Sclerosis Express EBV-Derived Proteins and \\ Activate Monocyte-Derived Macrophages \\ May F. Mrad, Esber S. Saba, Layane Nakib, et al. \\ Neurol Neuroimmunol Neuroinflamm 2021;8; \\ DOI 10.1212/NXI.0000000000001004
}

This information is current as of May 18, 2021

\begin{abstract}
Updated Information \& Services

References

Subspecialty Collections

Permissions \& Licensing

Reprints

including high resolution figures, can be found at:

http://nn.neurology.org/content/8/4/e1004.full.html

This article cites 39 articles, 9 of which you can access for free at: http://nn.neurology.org/content/8/4/e1004.full.html\#\#ref-list-1

This article, along with others on similar topics, appears in the following collection(s):

Multiple sclerosis

http://nn.neurology.org//cgi/collection/multiple_sclerosis its entirety can be found online at:

http://nn.neurology.org/misc/about.xhtml\#permissions

Information about ordering reprints can be found online: http://nn.neurology.org/misc/addir.xhtml\#reprintsus
\end{abstract}

Information about reproducing this article in parts (figures,tables) or in

Neurol Neuroimmunol Neuroinflamm is an official journal of the American Academy of Neurology.

Published since April 2014, it is an open-access, online-only, continuous publication journal. Copyright

Copyright $\odot 2021$ The Author(s). Published by Wolters Kluwer Health, Inc. on behalf of the American

Academy of Neurology.. All rights reserved. Online ISSN: 2332-7812.

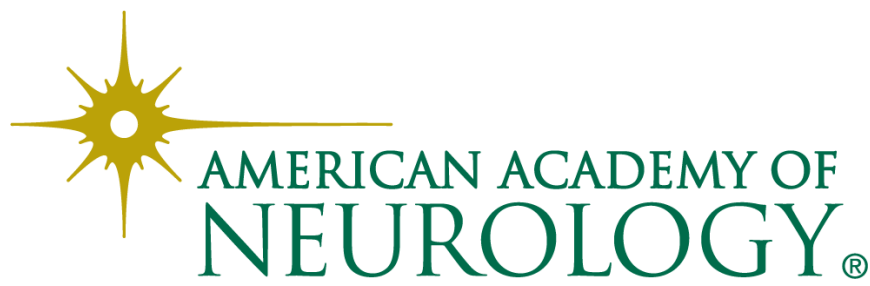

